\title{
Maps in dimension one with infinite entropy
}

\author{
Peter Hazard
}

\begin{abstract}
For each real $\alpha, 0 \leq \alpha<1$, we give examples of endomorphisms in dimension one with infinite topological entropy which are $\alpha$-Hölder; and for each real $p, 1 \leq p<\infty$, we also give examples of endomorphisms in dimension one with infinite topological entropy which are $(1, p)$-Sobolev. These examples are constructed within a family of endomorphisms with infinite topological entropy and which traverse all $\alpha$-Hölder and $(1, p)$-Sobolev classes. Finally, we also give examples of endomorphisms, also in dimension one, which lie in the big and little Zygmund classes, answering a question of M. Benedicks.
\end{abstract}

\section{Introduction}

\subsection{Background}

Adler, Konheim and McAndrew [1] defined the topological entropy of a continuous self-mapping of a compact metric space as an analogue of the Kolmogorov-Sinai metric entropy of a measure-preserving transformation of a measure space. Recall that the topological entropy of a continuous self-mapping is a non-negative real number, possibly infinite, which is invariant under topological conjugacy. (See [7], [16] for background.)

Determining what entropy values can be obtained by certain systems or classes of systems is a classical problem. The case of infinite topological entropy is of particular interest. Already in [1], an example of a map on a zero-dimensional space for which the topological entropy is infinite - a full shift on infinitely many symbols - was given. Later, in [10] further examples on symbol spaces were given which

This work has been partially supported by "Projeto Temático Dinâmica em Baixas Dimensões" FAPESP Grant 2011/16265-2, FAPESP Grant 2015/17909-7 and CAPES Projeto PVE CNPq 401020/2014-2.

Key words and phrases: entropy, Hölder classes, Sobolev classes, Zygmund classes. 2010 Mathematics Subject Classification: primary 37B40; secondary 37E05, 46E35, $26 \mathrm{~A} 16$. 
were strictly ergodic. A different approach was given in [9], where they constructed minimal homeomorphisms of the Cantor set, again with infinite entropy, without using methods from symbolic dynamics.

In [17], it was shown that even on smooth manifolds in higher dimensions there exist examples of continuous self-mappings with infinite topological entropy. In fact, a stronger statement was shown: for smooth compact manifolds of dimension two or greater, a generic homeomorphism (with respect to the uniform topology) has infinite topological entropy. In contrast, self-mappings with sufficient regularity or smoothness must have finite topological entropy. More precisely [12, Theorem 3.2 .9 ], if $f$ is a Lipschitz self-mapping of the compact metric space $(X, d)$ with finite Hausdorff dimension $D(X)$, we have the following inequality

$$
h_{\text {top }}(f) \leq D(X) \log ^{+} \operatorname{Lip}_{d}(f)
$$

where $h_{\text {top }}(f)$ denotes the topological entropy of $f$, and $\operatorname{Lip}_{d}(f)$ denotes the Lipschitz constant of $f$ with respect to the metric $d$. (See also [3], [11].)

In [5], an investigation was started into what occurs between $C^{0}$ and Lipschitz regularity, in the case of homeomorphisms on smooth compact manifolds. The notion of 'between' can be taken in several different directions. For compact subsets of the real line, for example, given $0 \leq \alpha<\beta<1$, if $C^{\alpha}$ denotes the space of $\alpha$-Hölder self-maps and $C^{Z}$ and $C^{\text {Lip }}$ denote the spaces of self-maps satisfying respectively the Zygmund and Lipschitz conditions $\left({ }^{1}\right)$ then

$$
C^{0} \supset C^{\alpha} \supset C^{\beta} \supset C^{Z} \supset C^{\text {Lip }} .
$$

Similarly, if $\mathbb{D} \mathbb{A} \mathbb{E}$ denotes the space of continuous self-maps which are differentiable (Lebesgue)-almost everywhere, $\mathbb{A} \mathbb{C}$ denotes the space of continuous self-maps which are absolutely continuous (with respect to Lebesgue), $\mathbb{B} \mathbb{V}$ denotes the space of continuous self-maps with bounded variation, and $\mathbb{W}^{1, p}, 1 \leq p \leq \infty$, denotes the space continuous self-maps satisfying the $W^{1, p}$-Sobolev condition, then

$$
C^{0}=U C \supset \mathbb{D} \mathbb{A} \supset \mathbb{B} \mathbb{V} \supset \mathbb{A} \simeq \mathbb{W}^{1,1} \supset \mathbb{W}^{1, p} \supset \mathbb{W}^{1, \infty} \simeq C^{\text {Lip }}
$$

With some care, (most of) these regularity classes, and the associated inclusions, can be extended to higher dimensions, and even to general smooth manifolds. In [5] an investigation into which values of entropy are possible in these two families of inclusions was initiated. It was shown that for any $\alpha \in[0,1)$ and $p \in[1, \infty)$, infinite entropy was not only possible, but a generic property in certain spaces (suitably topologised) of bi- $\alpha$-Hölder homeomorphisms, and of bi-(1, $p)$-Sobolev homeomorphisms, on smooth manifolds of dimension two or greater. Note that the result

$\left({ }^{1}\right)$ See [19] or Sections 1.4 and 3 below for definitions and basic properties. 
in [5] is only for the closure of bi-Lipschitz maps in the appropriate topology. M. Benedicks asked the following question:

Benedicks' Question: Is there a mapping in the big Zygmund class with infinite topological entropy? In the little Zygmund class?

Here we give an answer to these questions in the case of endomorphisms on compact one-manifolds. Specifically, we will work on the closed unit interval, but the generalisation to the circle case will follow immediately. (Note that homeomorphisms on compact one-manifolds must have zero entropy.)

Finally, we would like to bring the readers attention to the complementary result in [2], where it is shown - using the construction in [9] - that every Cantor minimal system can be realized as a minimal subsystem of a differentiable (though not continuously differentiable) endomorphism of the interval and, moreover, the derivative vanishes identically for this subsystem. In particular, the infinite entropy examples in [9] can be realised by differentiable interval endomorphisms. (This shows that, in particular, that in the class of differentiable maps the MargulisRuelle inequality fails quite dramatically.) We thank the referee for bringing this work to our attention.

\subsection{Summary of results}

First we construct examples of endomorphisms with infinite topological entropy lying in a Hölder or Sobolev class.

Theorem A. There exists a continuous one-parameter family of endomorphisms $f_{a} \in C([0,1],[0,1]), a \in(0,1]$, with the following properties

1. for all $a \in(0,1]$

a) all $f_{a}$ are topologically conjugate

b) $h_{\text {top }}\left(f_{a}\right)=+\infty$

c) $f_{a}$ is not expansive, $h$-expansive or asymptotically $h$-expansive

2. for $a=1$

a) $f_{a}$ has modulus of continuity $\omega(t)=t \log (1 / t)$

b) $f_{a}$ is in the Sobolev class $W^{1, p}$ for $1 \leq p<\infty$

c) Lebesgue measure is a measure of maximal entropy for $f_{a}$ (though there are at least countably many such measures)

3. for $a \in(0,1)$

a) $f_{a}$ is $C^{\alpha}$ if and only if $\alpha \leq a$.

b) $f_{a}$ is $W^{1, p}$ if and only if $p<(1-a)^{-1}$

c) Lebesgue measure is not preserved by $f_{a}$, but there exist measures of maximal entropy for $f_{a}$ which are absolutely continuous with respect to Lebesgue. 
Moreover, this family can be chosen so that each $f_{a}$ is topologically transitive.

We note that, for the specific examples considered here, half of the work is already done by Morrey's inequality: namely, if $f_{a}$ lies in $W^{1, p}$ then it automatically follows that $f_{a}$ is $C^{\alpha}$, where $\alpha=1-\frac{1}{p}$. However, we also give an explicit proof of Theorem $\mathrm{A}(3)(\mathrm{a})$. The reason being that our construction is made using piecewiseaffine horseshoes as 'model maps' from which the construction is made. If the model map which we start with is Hölder but not, for instance, differentiable almost everywhere, then our construction and estimates, suitably modified, still apply.

Remark 1.1. Similar examples were already constructed in [6]. However, the construction there made determining the possible conjugacy between different $f_{a}$ difficult. Our approach here simplifies this, while also giving the additional dynamical information in Theorem A above.

Following this we also construct examples of endomorphisms with infinite topological entropy satisfying the stronger Zygmund condition. Namely, the following is shown.

Theorem B. There exists $f \in C([0,1],[0,1])$ with the following properties

1. a) $f$ is not topologically conjugate to the examples in Theorem $A$

b) $h_{\text {top }}(f)=+\infty$

c) $f$ is not expansive, $h$-expansive or asymptotically $h$-expansive

2. $f$ satisfies the little Zygmund condition

Remark 1.2. Observe that Theorem B gives an affirmative answer to Benedicks' question stated above.

\subsection{Structure of the paper}

In Section 1.4, we set up notation and terminology for the rest of the paper, and recall some basic facts. In Section 2 we give a proof of Theorem A. First we construct the one-parameter family $f_{a}$ from which it will be clear that properties 1(a)-1(c) of Theorem A hold for all parameters $a \in(0,1]$. After this an elementary proof of properties 2(a)-2(c), i.e., for $a=1$, of Theorem A is given. Following this we prove some auxiliary propositions that are then used to prove properties $3(\mathrm{a})-3(\mathrm{~b})$. In Section 3, after recalling some basic definitions we give a proof of Theorem B. Finally, in Section 4 we end with some remarks and open problems. 


\subsection{Notation and terminology}

Throughout this article, we use the following notation. We denote the Euclidean norm in $\mathbb{R}$ by $|\cdot|_{\mathbb{R}}$ or $|\cdot|$ when there is no risk of ambiguity. We denote the Euclidean distance by $d(\cdot, \cdot)$. Given real-valued functions $f$ and $g$, defined on a subset of the real numbers accumulating on zero, we write $f(t)=O(g(t))$ if there exists $C>0$ such that, for $|t|$ sufficiently small, $|f(t)| \leq C|g(t)|$. We also write $f(t)=o(g(t))$ if $\lim _{t \rightarrow 0} \frac{|f(t)|}{|g(t)|}=0$.

\subsubsection{Hölder mappings}

Let $\alpha \in(0,1)$. Given a subset $\Omega$ of $\mathbb{R}$, let $C^{\alpha}(\Omega, \mathbb{R})$ denote the set of real-valued functions $f$ on $\Omega$ satisfying the $\alpha$-Hölder condition

$$
[f]_{\alpha, \Omega} \stackrel{\text { def }}{=} \sup _{x, y \in \Omega ; x \neq y} \frac{d(f(x), f(y))}{d(x, y)^{\alpha}}<\infty .
$$

When the domain of $f$ is clear we will write $[f]_{\alpha}$ instead of $[f]_{\alpha, \Omega}$. The $\operatorname{set} C^{\alpha}(\Omega, \mathbb{R})$ has a linear structure and $[\cdot]_{\alpha, \Omega}$ defines a semi-norm $\left({ }^{2}\right)$, which we call the $C^{\alpha}$-seminorm. Consequently

$$
\|f\|_{C^{\alpha}(\Omega, \mathbb{R})} \stackrel{\text { def }}{=}\|f\|_{C^{0}(\Omega, \mathbb{R})}+[f]_{\alpha, \Omega}
$$

defines a complete norm on $C^{\alpha}(\Omega, \mathbb{R})$.

For the same subset $\Omega$ of $\mathbb{R}$ let $C^{\operatorname{Lip}}(\Omega, \mathbb{R})$ denote the set of real-valued functions $f$ on $\Omega$ satisfying the Lipschitz condition

$$
[f]_{\operatorname{lip}, \Omega} \stackrel{\text { def }}{=} \sup _{x, y \in \Omega ; x \neq y} \frac{d(f(x), f(y))}{d(x, y)}<\infty .
$$

As before, this defines a semi-norm on the linear space $C^{\operatorname{Lip}}(\Omega, \mathbb{R})$. We denote the corresponding norm by $\|\cdot\|_{C^{\mathrm{Lip}}(\Omega, \mathbb{R})}$. Then, as above, this defines a Banach space structure on $C^{\operatorname{Lip}}(\Omega, \mathbb{R})$.

\subsubsection{Sobolev mappings}

Given an open subset $\Omega$ of $\mathbb{R}$, the Sobolev class $W^{1, p}(\Omega)$ consists of measurable functions $f: \Omega \rightarrow \mathbb{R}$ for which the first distributional partial derivative is defined and belongs to $L^{p}(\Omega)$. Then $W^{1, p}(\Omega)$ is a Banach space with respect to the norm

$$
\|u\|_{1, p}=\|u\|_{L^{p}}+\|D u\|_{L^{p}} .
$$

$\left({ }^{2}\right)$ This also induces a pseudo-distance which we will call the $C^{\alpha}$-pseudo-distance. 
Define the space

$$
\mathbb{W}^{1, p}(\Omega, \mathbb{R})=W^{1, p}(\Omega, \mathbb{R}) \cap C^{0}(\bar{\Omega}, \mathbb{R})
$$

For $f \in \mathbb{W}^{1, p}(\Omega, \mathbb{R})$ define

$$
[f]_{W^{1, p}, \Omega}=\left(\int_{\Omega}|D f(x)|^{p} d x\right)^{\frac{1}{p}} .
$$

Observe that $\mathbb{W}^{1, p}(\Omega, \mathbb{R})$ is a linear space and that $[\cdot]_{W^{1, p}, \Omega}$ defines a semi-norm which we call the $W^{1, p}$-semi-norm. Setting

$$
\|f\|_{\mathbb{W} 1, p(\Omega, \mathbb{R})}=\|f\|_{C^{0}(\bar{\Omega}, \mathbb{R})}+[f]_{W^{1, p}, \Omega},
$$

this defines a norm on $\mathbb{W}^{1, p}(\Omega, \mathbb{R})$ which is complete, and thus $\mathbb{W}^{1, p}(\Omega, \mathbb{R})$ is endowed with the structure of a Banach space.

\subsubsection{Topological entropy and expansivity}

Let $(X, d)$ be a compact metric space. Let $f$ be a continuous self-map of $(X, d)$. For each $n \in \mathbb{N}$ define the distance function

$$
d_{n}^{f}(x, y)=\max _{0 \leq k<n} d\left(f^{k}(x), f^{k}(y)\right) .
$$

Given sets $E, F \subset X$, we say that the set $E(n, \delta)$-spans the set $F$ with respect to $f$ if for any $x \in F$, there exists $y \in E$ such that $d_{n}^{f}(x, y)<\delta$. Let

$$
r_{f}(n, \delta ; F)=\min \{\# E: E(n, \delta) \text {-spans } F \text { with respect to } f\} .
$$

(Note that: (i) if $F$ is compact then $r_{f}(n, \delta ; F)<\infty$; (ii) $r_{f}(n, \delta ; F)$ increases as $\delta$ decreases.) For each compact set $K \subset X$, define $\left({ }^{3}\right)$

$$
r_{f}(\delta ; K)=\limsup _{n \rightarrow \infty} \frac{1}{n} \log r_{f}(n, \delta ; K)
$$

and

$$
h(f ; K)=\lim _{\delta \rightarrow 0} r_{f}(\delta ; K) .
$$

Since $X$ is compact we can define

$$
h(f, \delta)=h(f, \delta ; X) .
$$

$\left({ }^{3}\right)$ Here we depart from the notation originally due to Bowen [4]. 
The topological entropy of $f$ is defined by

$$
h_{\text {top }}(f)=\lim _{\delta \rightarrow 0} h(f, \delta)=\sup _{K} h(f ; K) .
$$

For each $\varepsilon>0$ and $x \in X$ define

$$
\Phi_{\varepsilon}(x)=\bigcap_{n \geq 0} f^{-n} B_{\varepsilon}\left(f^{n}(x)\right)=\left\{y: d\left(f^{n}(x), f^{n}(y)\right) \leq \varepsilon, \forall n \geq 0\right\} .
$$

Recall that $f$ is expansive if there exists $\varepsilon>0$ with the following property: given any $x, y \in X$, if $d\left(f^{k}(x), f^{k}(y)\right)<\varepsilon$, for all $k \in \mathbb{N}$, then $x=y$. Define

$$
h_{f}^{*}(\varepsilon)=\sup _{x \in X} h\left(f ; \Phi_{\varepsilon}(x)\right) .
$$

Then $f$ is $h$-expansive if $h_{f}^{*}(\varepsilon)=0$ for some $\varepsilon>0$; and is asymptotically $h$-expansive if $\lim _{\varepsilon \rightarrow 0} h_{f}^{*}(\varepsilon)=0$.

\section{Examples in Hölder and Sobolev classes}

We construct a family of endomorphisms $f_{a}$ of the unit interval, depending upon the parameter $a \in(0,1]$, such that each $f_{a}$ satisfies $h_{\text {top }}\left(f_{a}\right)=\infty$, it is not expansive, $h$-expansive or even asymptotically $h$-expansive, and such that all the $f_{a}$ are topologically conjugate. The main part of the work will then be in showing each $f_{a}$ has some intermediate regularity between $C^{0}$ and Lipschitz.

Remark 2.1. On compact one-manifolds, a theorem of Misiurewicz [14] states that positive topological entropy, and thus infinite topological entropy, must come from some iterate possessing a horseshoe. More precisely, if $h_{\text {top }}(f)>0$, then there exist sequences of positive integers $k_{n}$ and $s_{n}$ such that, for each $n, f^{k_{n}}$ possesses an $s_{n}$-branched horseshoe $\left({ }^{4}\right)$ and

$$
\lim _{n \rightarrow \infty} \frac{1}{k_{n}} \log s_{n}=h_{\mathrm{top}}(f) .
$$

Thus examples given below, which are constructed so that certain iterates possess horseshoes, are somehow indicative of the general case.

It will be useful to first consider an auxiliary family $g_{a, b}$ of interval maps defined as follows. First fix a positive integer $b$. Given an arbitrary interval $J$, let $A_{J}$ denote the unique orientation-preserving affine bijection from $J$ to $[0,1]$. Subdivide

$\left({ }^{4}\right)$ A map $g$ possesses an $s$-branched horseshoe if there is an interval $J$ with $s$ pairwise disjoint subintervals $J_{1}, J_{2}, \ldots, J_{s}$, such that $g\left(J_{j}\right) \subseteq J$ for $j=1,2, \ldots, s$. 
the interval $[0,1]$ into $b$ closed intervals $J_{b, 0}, J_{b, 1}, \ldots, J_{b, b-1}$ of equal length, ordered from left to right. Let $A_{b, k}=A_{J_{b, k}}$ for each $k=0,1, \ldots, b-1$. Let $\nu$ denote the unique orientation-reversing affine bijection of $[0,1]$ to itself. For each $k=0,1, \ldots, b-1$, define

$$
g_{1, b}(x)=\nu^{k} \circ A_{b, k}(x), \quad \forall x \in J_{b, k}
$$

More explicitly

$$
g_{1, b}(x)=\left\{\begin{array}{lll}
b x-k & x \in J_{b, k}, & k \text { even } \\
(k+1)-b x & x \in J_{b, k}, & k \text { odd }
\end{array} .\right.
$$

Observe that $g_{1, b}$ is continuous on $[0,1]$. Also, $[0,1]$ possesses a $g_{1, b}$-invariant subset on which $g_{1, b}$ acts as the unilateral shift on $b$ symbols. In fact, $h_{\text {top }}\left(g_{1, b}\right)=\log b$ (see e.g. [12, Section 3.2.c]).

Next, take a continuous one-parameter family $\varphi_{a}, a \in(0,1]$, of orientationpreserving homeomorphisms of $[0,1]$, with $\varphi_{1}=\mathrm{id}$, and define

$$
g_{a, b}=\varphi_{a} \circ g_{1, b} \circ \varphi_{a}^{-1}
$$

For example, we could take $\varphi_{a}$ equal to $q_{a}(x)=x^{a}$, the power function of exponent $a$. (Observe that in this case $g_{a, b}$ is $C^{a}$ but not $C^{\alpha}$ for any $\alpha>a$, provided that $b \geq 2$.) Then $g_{a, b}$ is continuous on $[0,1]$. As topological entropy is invariant under topological conjugacy, we also have $h_{\text {top }}\left(g_{a, b}\right)=\log b$, for each $a \in(0,1]$ and each positive integer $b$. We call $b$ the number of branches of $g_{a, b}$ and $a$ the order of singularity.

We now define the family $f_{a}$ as follows. For each positive integer $n$ define the interval $I_{n}=\left(2^{-n}, 2^{-n+1}\right]$ and let $f_{a}$ be given by

$$
f_{a}(x)=\left\{\begin{array}{ll}
A_{I_{n}}^{-1} \circ g_{a, 2 n+1} \circ A_{I_{n}}(x) & x \in I_{n}, n=1,2, \ldots \\
0 & x=0
\end{array} .\right.
$$

Observe that, since $g_{a, 2 n+1}$ fixes the endpoints of $[0,1]$ and is continuous, the map $f_{a}$ is also continuous. Also, since, for each fixed $b$, all the functions $g_{a, b}, a \in(0,1]$, are topologically conjugate, it follows that all the functions $f_{a}, a \in(0,1]$, are also all topologically conjugate. Namely, $f_{a}=\psi_{a}^{-1} \circ f_{1} \circ \psi_{a}$ where

$$
\psi_{a}(x)=A_{I_{n}}^{-1} \circ \varphi_{a} \circ A_{I_{n}}(x) \quad \forall x \in I_{n}, \forall n \in \mathbb{N} .
$$

Notice that the closure of each interval $I_{n}$ is totally invariant. Since the topological entropy of a map is the supremum of the topological entropy of its restriction to all closed invariant subsets, since topological entropy is invariant under topological 
conjugacy (see e.g. [12, Section 3.1.b]) and, as was stated above, $h_{\text {top }}\left(g_{a, b}\right)=\log b$ for all $b$, it follows that

$$
h_{\mathrm{top}}\left(f_{a}\right) \geq \sup _{n} h_{\mathrm{top}}\left(\left.f_{a}\right|_{I_{n}}\right)=\sup _{n} h_{\mathrm{top}}\left(g_{a, 2 n+1}\right)=+\infty .
$$

Next, observe that, as $f_{a}$ has arbitrarily small invariant subsets (namely the intervals $\left.I_{n}\right)$ the function $f_{a}$ cannot be expansive. In fact, since $h\left(f_{a}, I_{n}\right)=\log (2 n+1)$ for each $n$, it follows that

$$
\lim _{\varepsilon \rightarrow 0} h_{f_{a}}^{*}(\varepsilon) \geq \lim _{n \rightarrow \infty} h\left(f_{a} ; I_{n}\right)=+\infty .
$$

Thus $f_{a}$ is neither $h$-expansive nor asymptotically $h$-expansive. Therefore properties 1(a) $-1(\mathrm{c})$ of Theorem A hold.

Remark 2.2. That $f_{a}$ is not asymptotically $h$-expansive could also be shown using topological conditional entropy in the following way. By [13, Proposition 3.3] infinite topological entropy $h_{\text {top }}\left(f_{a}\right)$ implies infinite topological conditional entropy $h^{*}\left(f_{a}\right)$. However, by [13, Corollary $\left.2.1(\mathrm{~b})\right] f_{a}$ is asymptotically $h$-expansive if and only if $h^{*}\left(f_{a}\right)=0$.

Proof of Theorem A 2(a)-2(c). For each positive integer $n$, define the subintervals $I_{n, k}=A_{I_{n}}^{-1}\left(J_{2 n+1, k}\right)$ of $I_{n}$ for $k=0,1, \ldots, 2 n$. These denote the maximal closed subintervals of $I_{n}$ on which $f_{a}$ is monotone.

(a) Take distinct points $x, y \in[0,1]$. There are three cases to consider.

$\left(x \in I_{n}, y \in I_{m}, n>m\right)$ Since $I_{m}$ and $I_{n}$ are both $f$-invariant, $f(x) \in I_{n}$ and $f(y) \in$ $I_{m}$. Moreover, observe that

$$
|f(x)-f(y)| \leq\left|2^{-n}-2^{-m+1}\right|<2^{-m+1}
$$

together with

$$
|x-y| \geq\left|2^{-n+1}-2^{-m}\right| \geq 2^{-m-1}
$$

implies that

$$
\frac{|f(x)-f(y)|}{\omega(|x-y|)} \leq \frac{2^{-m+1}}{2^{-m-1} \log 2^{m+1}}=\frac{4}{(m+1) \log 2} .
$$

$\left(x=0, y \in I_{m}\right)$ Applying the same argument as in the previous case and observing that $f(x)=x=0$ we find that

$$
\frac{|f(x)-f(y)|}{\omega(|x-y|)} \leq \frac{2^{-m+1}}{2^{-m-1} \log 2^{m+1}}=\frac{4}{(m+1) \log 2} \leq \frac{2}{\log 2} .
$$




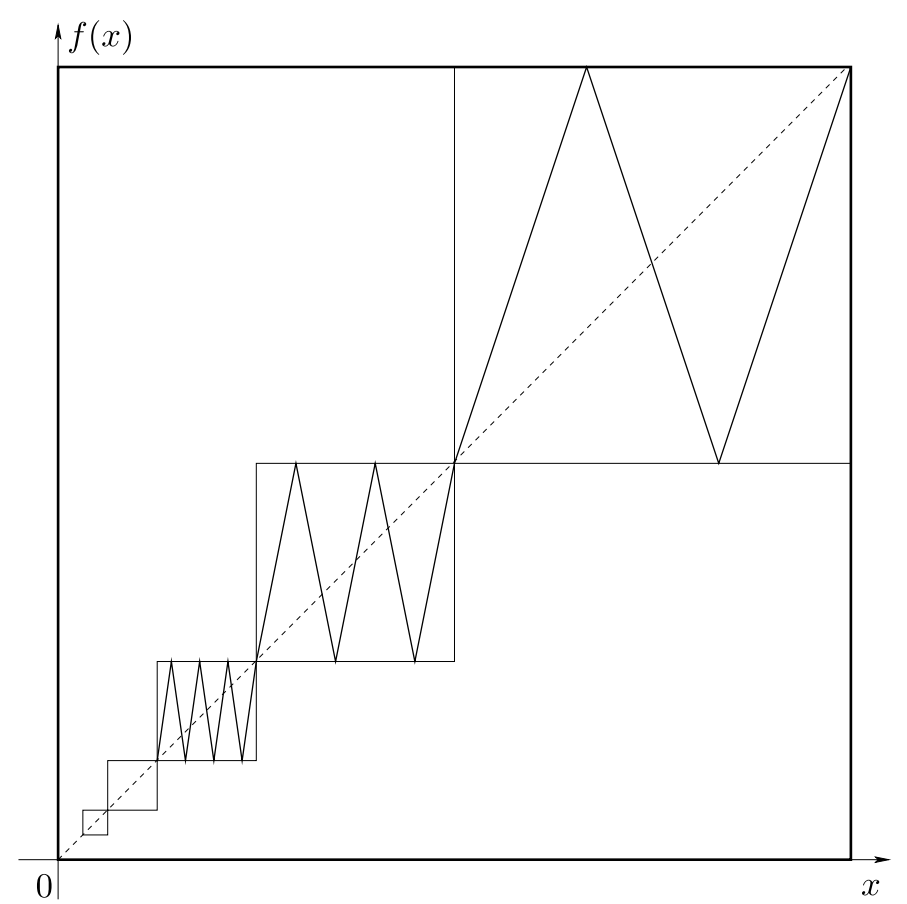

Figure 1. The graph of the Hölder interval endomorphism $f_{1}$ with infinite topological entropy.

$\left(x \in I_{n}, y \in I_{m}, n=m\right)$ If $x$ and $y$ do not lie in the same branch of $\left.f\right|_{I_{k}}$, then there exists $y^{\prime}$, in the same branch as $x$, satisfying $f(y)=f\left(y^{\prime}\right)$ and $|x-y|>\left|x-y^{\prime}\right|$. Moreover,

$$
\left|x-y^{\prime}\right| \leq \frac{\left|I_{m}\right|}{2 m+1}=\frac{1}{2^{m}(2 m+1)}
$$

Thus

$$
\begin{aligned}
\frac{|f(x)-f(y)|}{\omega(|x-y|)} \leq \frac{\left|f(x)-f\left(y^{\prime}\right)\right|}{\omega\left(\left|x-y^{\prime}\right|\right)} & =\frac{(2 m+1)\left|x-y^{\prime}\right|}{\left|x-y^{\prime}\right| \log \left(\left|x-y^{\prime}\right|-1\right)} \\
& \leq \frac{2 m+1}{\log 2^{m}(2 m+1)} \\
& \leq \frac{2}{\log 2}+1 .
\end{aligned}
$$

In each of these cases, for $x, y \in[0,1], x \neq y$,

$$
\frac{|f(x)-f(y)|}{\omega(|x-y|)} \leq \frac{2}{\log 2}+1
$$


and hence $f$ has modulus of continuity $\omega$, which completes the proof of part (i).

(b) Observe that $f$ is differentiable except at the endpoints of the intervals $I_{k, l}$. Hence

$$
\left|f^{\prime}\right|_{I_{n}} \mid=\frac{\left|I_{n}\right|}{\left|I_{n, k}\right|}=2 n+1 \text {. }
$$

Therefore, as the $I_{n}$ form a measurable partition of $[0,1]$,

$$
\begin{aligned}
\int_{[0,1]}\left|f^{\prime}\right|^{p} d x=\sum_{n=1}^{\infty} \int_{I_{n}}\left|f^{\prime}\right|^{p} d x & =\sum_{n=1}^{\infty}(2 n+1)^{p} \int_{I_{n}} d x \\
& =\sum_{n=1}^{\infty}(2 n+1)^{p} 2^{-k} \\
& \leq \sum_{n=1}^{\infty} n^{p} 2^{-(n-1) / 2} \\
& =2^{1 / 2} \sum_{n=1}^{\infty} n^{p} 2^{-n / 2}
\end{aligned}
$$

However, this last series is convergent. Consequently the Sobolev norm of $f$ is finite and hence $f \in W^{1, p}([0,1])$.

(c) First note that as $g_{1, b}$ preserves Lebesgue measure $\mu$ for each $b$, it follows trivially that Lebesgue measure is invariant under $f_{1}$. Since $h_{\mu}\left(g_{1, b}\right)=\log b$, it also follows that Lebesgue measure is a measure of maximal entropy. Hence the theorem is shown.

For each positive integer $n$, by performing the same construction as above but just on the union of the intervals $I_{n}, I_{n+1}, \ldots$ we also get the following corollary.

Corollary 2.1. There exists a sequence $f_{n} \in C^{0}([0,1],[0,1])$ satisfying properties 2(a)-2(c) in Theorem $A$ above and with the additional property that $\lim _{n \rightarrow \infty} f_{n}=$ id where convergence is taken

- in the $C^{\alpha}$-topology for any $\alpha \in(0,1)$,

- in the $W^{1, p}$-topology for any $p \in[1, \infty)$.

We recall that maps with modulus of continuity $t \log (1 / t)$ are in the Hölder class $C^{\alpha}$ for every $\alpha \in[0,1)$, but they are not necessarily Lipschitz. Moreover, the map $f_{1}$ is a $C^{\alpha}$-limit of piecewise-affine maps. Hence it lies in the $C^{\alpha}$-boundary of the space of Lipschitz maps. When $a \neq 1$, the map $f_{a}$ does not satisfy this property. The proof of Theorem A 3(a)-3(b) could be made using the argument presented above in the proof of properties $2(\mathrm{a})-2(\mathrm{~b})$ of Theorem A. However, we give a different 
proof below. For that we need the following gluing principle, which is essentially an application of Jensen's inequality.

Proposition 2.1. (Gluing Principle) Let $\omega$ be a continuous, monotone-increasing function, locally concave at $\omega(0)=0$. Let $f$ be a continuous self-mapping of the compact interval I. Let $I_{1}, I_{2}, \ldots$ denote a collection of closed intervals with pairwise disjoint interiors, covering $I$, and with the property that $\left.f\right|_{I_{k}}$ has modulus of continuity $\omega$, for all $k$. Let $C_{k}$ denote the $\omega$-semi-norm of $\left.f\right|_{I_{k}}$.

(i) If $\sum_{k=1}^{\infty} C_{k}<\infty$ then $f$ has modulus of continuity $\omega$ with $\omega$-semi-norm bounded by $C=\sum_{k=1}^{\infty} C_{k}$.

(ii) If $\sup _{k} C_{k}<\infty$ and $\left.f\right|_{\partial I_{k}}=\mathrm{id}$ for all $k$, then $f$ has modulus of continuity $\omega$ with $\omega$-semi-norm bounded by $C=\frac{\operatorname{diam}(I)}{\omega(\operatorname{diam}(I))}+2 \sup _{k} C_{k}$.

Proof. For notational simplicity, assume that the intervals $I_{k}$ are ordered from left to right. This does not affect the proof, but simplifies indexing.

Case (i). Take $x, y \in I$. Assume that $x<y$. Then there exist integers $m<n$ such that $x \in I_{m}, y \in I_{n}$. Consequently

$$
x_{m}=x<x_{m+1}<\ldots<x_{n}<y=x_{n+1},
$$

where the points $x_{m+1}, x_{m+2} \ldots, x_{n}$ denote the left endpoints of the respective intervals $I_{m+1}, I_{m+2}, \ldots, I_{n}$. Let $C_{k}$ denote the $\omega$-semi-norm of $\left.f\right|_{I_{k}}$, that is

$$
C_{k}=\sup _{z \neq w \in I_{k}} \frac{d(f(z), f(w))}{\omega(d(z, w))} .
$$

It follows that

$$
d(f(x), f(y)) \leq \sum_{k=m}^{n} d\left(f\left(x_{k}\right), f\left(x_{k+1}\right)\right) \leq \sum_{k=m}^{n} C_{k} \omega\left(d\left(x_{k}, x_{k+1}\right)\right)
$$

However, since $\Lambda$ is concave, Jensen's inequality implies that

$$
\begin{aligned}
\frac{\sum_{k=m}^{n} C_{k} \omega\left(d\left(x_{k}, x_{k+1}\right)\right)}{\sum_{k=m}^{n} C_{k}} & \leq \omega\left(\frac{\sum_{k=m}^{n} C_{k} d\left(x_{k}, x_{k+1}\right)}{\sum_{k=m}^{n} C_{k}}\right) \\
& \leq \omega\left(\max _{m \leq k \leq n} C_{k} \cdot \frac{\sum_{k=m}^{n} d\left(x_{k}, x_{k+1}\right)}{\max _{m \leq k \leq n} C_{k}}\right) \\
& =\omega(d(x, y)) .
\end{aligned}
$$

where, for the last equality we have used that the points $x_{k}$ are in the real line, placed in increasing order. Combining inequalities (2.25) with (2.28) together with the hypothesis that $\sum_{k=1}^{\infty} C_{k}<\infty$ gives the result by taking the supremum over all possible $x$ and $y$. 
Case (ii). Take $x, y$ and $x_{m+1}, \ldots, x_{n}$ as before. Then

$$
\begin{aligned}
d(f(x), f(y)) & \leq d\left(f(x), f\left(x_{m+1}\right)\right)+d\left(f\left(x_{m+1}\right), f\left(x_{n}\right)\right)+d\left(f\left(x_{n}\right), f(y)\right) \\
& \leq C_{m} \omega\left(d\left(x, x_{m+1}\right)\right)+d\left(x_{m+1}, x_{n}\right)+C_{n} \omega\left(d\left(x_{n}, y\right)\right) \\
& \leq\left(2 \sup _{k} C_{k}+\frac{\operatorname{diam}(I)}{\omega(\operatorname{diam}(I))}\right) \omega(d(x, y)) .
\end{aligned}
$$

As this holds for all $x$ and $y$, it follows that $f$ has modulus of continuity $\omega$, with $\omega$-semi-norm bounded by $2 \sup _{k} C_{k}+\operatorname{diam}(I) / \omega(\operatorname{diam}(I))$, as required.

We will also need the following estimates for the map $g_{a, b}$ with respect to the Hölder and Sobolev semi-norms, in the case when $\varphi_{a}$ is a general concave orientation-preserving homeomorphism.

Lemma 2.1. (Auxiliary Lemma) Let $g_{a, b}$ be defined as above, where $\varphi_{a}$ is an arbitrary concave, orientation-preserving homeomorphism, so that it possesses an extension to $\left[0,1+\frac{1}{b}\right]$, which is also concave and a homeomorphism onto its image. Then

$$
\left[g_{a, b}\right]_{C^{\alpha},[0,1]} \leq\left[\varphi_{a}\right]_{C^{\alpha},[0,1]} \cdot b^{\alpha+1} \int_{\left[\frac{1}{b}, 1+\frac{1}{b}\right]}\left|\left(\varphi_{a}^{-1}\right)^{\prime}(t)\right|^{\alpha} d t
$$

and

$$
\left[g_{a, b}\right]_{W^{1, p},[0,1]}^{p} \leq\left[\varphi_{a}\right]_{W^{1, p},[0,1]} \cdot b^{p}\left(\int_{[0,1]}\left|\frac{t}{g_{1, b}(t)}\right|^{\frac{p^{2}(1-a)}{p-1}} d t\right)^{1-\frac{1}{p}}
$$

Remark 2.3. As $\varphi_{a}$ is monotone increasing it follows by Lebesgue's Last Theorem that it is differentiable Lebesgue-almost everywhere (see, e.g. [15]). Since it is concave it follows from Alexandrov's theorem that is it also twice-differentiable Lebesgue-almost everywhere [8, Section 6.4].

Proof. Before starting the proof, we introduce the following notation and make the following comments. For any $t \in[0,1]$ we use the notation

$$
t^{\prime}=\varphi_{a}^{-1}(t), \quad t^{\prime \prime}=g_{1, b}\left(\varphi_{a}^{-1}(t)\right) .
$$

First consider the Hölder estimate. Take $k \in\{0,1, \ldots, b-1\}$. Let $x, y \in J_{b, k}$ be arbitrary distinct points. Then, by telescoping the $a$-Hölder difference quotient, and observing that $g_{1, b}$ is affine, we find that

$$
\frac{\left|g_{a, b}(x)-g_{a, b}(y)\right|}{|x-y|^{\alpha}}=b^{\alpha} \frac{\left|\varphi_{a}\left(x^{\prime \prime}\right)-\varphi_{a}\left(y^{\prime \prime}\right)\right|}{\left|x^{\prime \prime}-y^{\prime \prime}\right|^{a}}\left(\frac{\left|\varphi_{a}^{-1}(x)-\varphi_{a}^{-1}(y)\right|}{|x-y|}\right)^{\alpha} .
$$


Observe that $x^{\prime \prime}$ and $y^{\prime \prime}$ take values throughout $[0,1]$. Therefore

$$
\frac{\left|\varphi_{a}\left(x^{\prime \prime}\right)-\varphi_{a}\left(y^{\prime \prime}\right)\right|}{\left|x^{\prime \prime}-y^{\prime \prime}\right|^{\alpha}} \leq\left[\varphi_{a}\right]_{C^{\alpha},[0,1]} .
$$

Next, trivially $x$ and $y$ take values throughout $J_{b, k}=\left[\frac{k}{b}, \frac{k+1}{b}\right]$. Therefore, since the function $\varphi_{a}^{-1}(t)$ is convex and increasing on the positive real line (and thus difference quotients on $J_{b, k}$ are maximised by the derivative at the right endpoint $\partial^{+} J_{b, k}$ ),

$$
\left(\varphi_{a}^{-1}\right)^{\prime}\left(\partial^{-} J_{b, k}\right) \leq \frac{\left|\varphi_{a}^{-1}(x)-\varphi_{a}^{-1}(y)\right|}{|x-y|} \leq\left(\varphi_{a}^{-1}\right)^{\prime}\left(\partial^{+} J_{b, k}\right) .
$$

Consequently, by Proposition 2.1(i), together with the fact that $\left(\varphi_{a}^{-1}\right)^{\prime}$ is increasing on the positive real line (so $\left(\varphi_{a}^{-1}\right)^{\prime}$ is minimised on $J_{b, k}$ by its value at the left endpoint $\left.\partial^{-} J_{b, k}=\frac{k}{b}\right)$ we have

$$
\begin{aligned}
{\left[g_{a, b}\right]_{C^{\alpha},[0,1]} } & \leq \sum_{k=0}^{b-1}\left[g_{a, b}\right]_{C^{\alpha}, J_{b, k}} \\
& \leq\left[\varphi_{a}\right]_{C^{\alpha},[0,1]} \cdot b^{\alpha+1} \sum_{k=0}^{b-1}\left|\left(\varphi_{a}^{-1}\right)^{\prime}\left(\frac{k+1}{b}\right)\right|^{\alpha} \cdot \frac{1}{b} \\
& =\left[\varphi_{a}\right]_{C^{\alpha},[0,1]} \cdot b^{\alpha+1} \sum_{k=0}^{b-1}\left|\left(\varphi_{a}^{-1}\right)^{\prime}\left(\partial^{-} J_{b, k+1}\right)\right|^{\alpha} \cdot\left|J_{b, k+1}\right| \\
& \leq\left[\varphi_{a}\right]_{C^{\alpha},[0,1]} \cdot b^{\alpha+1} \int_{\left[\frac{1}{b}, 1+\frac{1}{b}\right]}\left|\left(\varphi_{a}^{-1}\right)^{\prime}(t)\right|^{\alpha} d t .
\end{aligned}
$$

Next, consider the Sobolev case. Observe that $g_{a, b}$ is differentiable everywhere except a finite set of points. More precisely, $g_{a, b}$ has breaks at exactly the endpoints of $\varphi_{a}\left(J_{b, k}\right)$ for $k=0,1, \ldots, b-1$. By the chain rule, at Lebesgue almost every point $x$ we have

$$
\begin{aligned}
\left|g_{a, b}^{\prime}(x)\right| & =\mid \varphi_{a}^{\prime}\left(g_{1, b}\left(\varphi_{a}^{-1}(x)\right)|| g_{1, b}^{\prime}\left(\varphi_{a}^{-1}(x)\right)||\left(\varphi_{a}^{-1}\right)^{\prime}(x) \mid\right. \\
& =b\left(\frac{\left|g_{1, b}\left(\varphi_{a}^{-1}(x)\right)\right|}{\left|\varphi_{a}^{-1}(x)\right|}\right)^{a-1} .
\end{aligned}
$$

Thus, by the change of variable formula for integrals

$$
\begin{aligned}
{\left[g_{a, b}\right]_{W^{1, p}, \varphi_{a}\left(J_{b, k}\right)}^{p} } & =\int_{\varphi_{a}\left(J_{b, k}\right)}\left|g_{a, b}^{\prime}(x)\right|^{p} d \mu(x) \\
& =b^{p} \int_{\varphi_{a}\left(J_{b, k}\right)}\left|\frac{g_{1, b}\left(\varphi_{a}^{-1}(x)\right)}{\varphi_{a}^{-1}(x)}\right|^{p(a-1)} d \mu(x)
\end{aligned}
$$




$$
=b^{p} \int_{J_{b, k}}\left|\frac{g_{1, b}(t)}{t}\right|^{p(a-1)}\left|\varphi_{a}^{\prime}(t)\right| d \mu(t) .
$$

Therefore

$$
\left[g_{a, b}\right]_{W^{1, p},[0,1]}^{p}=\sum_{k=0}^{b-1}\left[g_{a, b}\right]_{W^{1, p}, J_{b, k}}^{p}=b^{p} \int_{[0,1]}\left|\frac{t}{g_{1, b}(t)}\right|^{p(1-a)}\left|\varphi_{a}^{\prime}(t)\right| d \mu(t) .
$$

Therefore applying Hölder's inequality gives the result.

Corollary 2.2. Let $b \geq 2$. Let $\varphi_{a}=q_{a}$ for each $a \in[0,1]$. Then there exist positive real numbers $C(a, \alpha)$ and $K(a, p)$, depending only upon $a$ and $\alpha$ and upon $a$ and $p$ respectively, such that we have the following for each $a \in[0,1)$ :

(i) $g_{a, b}$ is $C^{\alpha}$ for all $\alpha \leq a$ and

$$
\left[g_{a, b}\right]_{C^{\alpha},[0,1]} \leq C(a, \alpha) b^{\alpha+1}
$$

(ii) $g_{a, b}$ is $W^{1, p}$ for all $p<(1-a)^{-1}$ and

$$
\left[g_{a, b}\right]_{W^{1, p},[0,1]}^{p} \leq K(a, p) b^{p(1-a)+1} .
$$

Proof. (i) When $\varphi_{a}=q_{a}$ we find that $q_{a, b}$ is locally of the form $|x|^{a}$ about any $g_{a, b}$-preimage of 0 except 0 itself. (Observe that, as $b \geq 2$, such a preimage exists.) Therefore $g_{a, b}$ cannot be $C^{\alpha}$ for any $\alpha>a$. For $\alpha \leq a$, by inequality (2.32),

$$
\left[g_{a, b}\right]_{C^{\alpha},[0,1]} \leq\left[\varphi_{a}\right]_{C^{\alpha},[0,1]} \frac{b^{\alpha+1} a^{1-\alpha}}{\alpha(1-a)+a}\left(\left(1+\frac{1}{b}\right)^{\alpha\left(\frac{1}{a}-1\right)+1}-\left(\frac{1}{b}\right)^{\alpha\left(\frac{1}{a}-1\right)+1}\right)
$$

and thus there exists an extended positive real number $C(a, \alpha)$, depending upon $a$ and $\alpha$ only, such that $C(a, \alpha)$ is finite for $0 \leq \alpha \leq a$, and is infinite for $a<\alpha \leq 1$, and for which

$$
\left[g_{a, b}\right]_{C^{\alpha},[0,1]} \leq C(a, \alpha) b^{\alpha+1} .
$$

(ii) First, in the special case when $k=0$,

$$
\left[g_{a, b}\right]_{W^{1, p}, \varphi_{a}\left(J_{b, 0}\right)}^{p}=b^{p} \int_{0}^{\frac{1}{b}}\left|\frac{1}{b}\right|^{p(1-a)} \varphi_{a}^{\prime}(t) d t=b^{a(p-1)} .
$$

In the general case, applying the standard $L^{1}$-estimate to inequality (2.33) gives

$$
\begin{gathered}
b^{p} \min _{J_{b, k}}\left|\varphi_{a}^{\prime}\right| \int_{J_{b, k}}\left|\frac{t}{g_{1, b}(t)}\right|^{p(1-a)} d t \leq\left[g_{a, b}\right]_{W^{1, p}, \varphi_{a}\left(J_{b, k}\right)}^{p} \\
\leq b^{p} \max _{J_{b, k}}\left|\varphi_{a}^{\prime}\right| \int_{J_{b, k}}\left|\frac{t}{g_{1, b}(t)}\right|^{p(1-a)} d t .
\end{gathered}
$$


But

$$
\int_{J_{b, k}}\left|\frac{t}{g_{1, b}(t)}\right|^{p(1-a)} d t=\left\{\begin{array}{l}
\int_{J_{b, k}}\left|\frac{t}{b t-k}\right|^{p(1-a)} d t \quad k \text { even } \\
\int_{J_{b, k}}\left|\frac{t}{k+1-b t}\right|^{p(1-a)} d t \quad k \text { odd }
\end{array}\right.
$$

Making an appropriate change of variables this can also be written in the form

$$
\int_{J_{b, k}}\left|\frac{t}{g_{1, b}(t)}\right|^{p(1-a)} d t=\left\{\begin{array}{ll}
\int_{[0,1]}\left|1+\frac{k}{u}\right|^{p(1-a)} b^{1-p(1-a)} d u & k \text { even } \\
\int_{[0,1]}\left|1-\frac{k+1}{u}\right|^{p(1-a)} b^{1-p(1-a)} d u & k \text { odd }
\end{array} .\right.
$$

Combining with (2.51), this shows that $g_{a, b}$ is not $W^{1, p}$ for $p \geq(1-a)^{-1}$. For $p<$ $(1-a)^{-1}$, since the power function $t^{\sigma}$, where $\sigma=p(1-a)$, is concave we find that

$$
\begin{aligned}
\int_{[0,1]}\left(1+\frac{k}{u}\right)^{\sigma} d u & \leq k^{\sigma} \int_{[0,1]} u^{-\sigma} d u+\sigma k^{\sigma-1} \int_{[0,1]} u^{1-\sigma} d u \\
& =\frac{k^{\sigma}}{1-\sigma}+\frac{\sigma k^{\sigma-1}}{2-\sigma}
\end{aligned}
$$

while

$$
\int_{[0,1]}\left(\frac{k+1}{u}-1\right)^{\sigma} d u \leq \int_{[0,1]}\left(\frac{k+1}{u}\right)^{\sigma} d u=\frac{(k+1)^{\sigma}}{1-\sigma} .
$$

Therefore, by (2.51) we arrive at the following inequalities

(2.57) $\left[g_{a, b}\right]_{W^{1, p}, \varphi_{a}\left(J_{b, k}\right)}^{p} \leq\left\{\begin{array}{ll}a\left(\frac{b}{k}\right)^{1-a} k^{p(1-a)}\left(\frac{1}{1-p(1-a)}+\frac{p(1-a)}{k(2-p(1-a))}\right) & k \text { even } \\ a\left(\frac{b}{k}\right)^{1-a}(k+1)^{p(1-a) \frac{1}{1-p(1-a)}} & k \text { odd }\end{array}\right.$.

Consequently, there exist positive real numbers $K_{0}(a, p)$ and $K(a, p)$, depending upon $a$ and $p$ only, such that

$$
\begin{aligned}
{\left[g_{a, b}\right]_{W^{1, p},[0,1]}^{p} } & =\sum_{k=0}^{b-1}\left[g_{a, b}\right]_{W^{1, p}, \varphi_{a}\left(J_{k, b}\right)}^{p} \\
& \leq K_{0}(a, p) b^{1-a} \sum_{k=0}^{b-1} k^{(p-1)(1-a)} \\
& \leq K(a, p) b^{p(1-a)+1}
\end{aligned}
$$

This completes the proof. 
Proof of Theorem A 3(a)-3(c). (a) Since the function $g_{a, b}$ is not $C^{\alpha}$ for any $\alpha>a$, and as the $\alpha$-Hölder condition is preserved under affine rescaling, it follows that $f_{a}$ is also not $C^{\alpha}$ for any $\alpha>a$. Let us show that $f_{a}$ lies in $C^{a}$. By the Hölder rescaling principle [5, Proposition 2.2]

$$
\begin{aligned}
{\left[f_{a}\right]_{C^{a}, I_{n}} } & \leq\left[A_{I_{n}}^{-1}\right]_{\operatorname{Lip}}\left[g_{a, 2 n+1}\right]_{C^{a},[0,1]}\left[A_{I_{n}}\right]_{\mathrm{Lip}}^{a} \\
& =\left|I_{n}\right|^{1-a}\left[g_{a, 2 n+1}\right]_{C^{a},[0,1]} \\
& \leq 2^{-n(1-a)} C(a, a)(2 n+1)^{1+a} .
\end{aligned}
$$

By the Proposition 2.1(ii), with $\omega(x)=x^{a}$, since $f_{a}$ fixed the endpoints of $I_{n}$ for each $n$, we find that

$$
\left[f_{a}\right]_{C^{a},[0,1]} \leq \sup _{n}\left[f_{a}\right]_{C^{a}, I_{n}} \leq C(a, a) \sup _{n} 2^{-n(1-a)}(2 n+1)^{1+a}<\infty
$$

where, for the second inequality, we used Corollary 2.2(i).

(b) Since $\left.f_{a}\right|_{I_{n}}=A_{I_{n}}^{-1} \circ g_{a, 2 n+1} \circ A_{I_{n}}$ an affine rescaling of a map differentiable Lebesgue-almost everywhere we find that, for Lebesgue-almost every $x \in I_{n}$,

$$
\left|f_{a}(x)\right|=\left|g_{a, 2 n+1}^{\prime}\left(A_{I_{n}}(x)\right)\right| .
$$

This, together with the change of variables formula for integrals and the observation that $\left|A_{I_{n}}^{\prime}\right|=\left|I_{n}\right|^{-1}$, gives

$$
\begin{aligned}
{\left[f_{a}\right]_{W^{1, p}, I_{n}}^{p} } & =\int_{I_{n}}\left|f_{a}^{\prime}(x)\right|^{p} d x \\
& =\left|I_{n}\right| \int_{I_{n}}\left|g_{a, 2 n+1}^{\prime}\left(A_{I_{n}}(x)\right)\right|^{p}\left|A_{I_{n}}^{\prime}(x)\right| d x \\
& =\left|I_{n}\right| \int_{A_{I_{n}}\left(I_{n}\right)}\left|g_{a, 2 n+1}^{\prime}(u)\right|^{p} d u \\
& \leq\left|I_{n}\right|\left[g_{a, 2 n+1}\right]_{W^{1, p},[0,1]}^{p} .
\end{aligned}
$$

This, together with Corollary 2.2(ii), implies that

$$
\begin{aligned}
{\left[f_{a}\right]_{W^{1, p},[0,1]}^{p} } & =\sum_{n=1}^{\infty}\left[f_{a}\right]_{W^{1, p}, I_{n}}^{p} \\
& \leq \sum_{n=1}^{\infty}\left|I_{n}\right|\left[g_{a, 2 n+1}\right]_{W^{1, p},[0,1]}^{p} \\
& \leq \sum_{n=1}^{\infty} 2^{-n} K(a, p)(2 n+1)^{p(1-a)+1}
\end{aligned}
$$

This last series is convergent. Thus $f_{a}$ is $W^{1, p}$ for $1 \leq p<(1-a)^{-1}$, as required. 
(c) Observe that $\varphi_{a}$ and $\varphi_{a}^{-1}$ are $C^{a}$, but they are not $C^{\alpha}$ for any $\alpha>a$. By the Hölder rescaling principle [5, Proposition 2.2]

$$
\left[\psi_{a}\right]_{C^{\alpha}, I_{n}} \leq\left[A_{I_{n}}^{-1}\right]_{\operatorname{Lip}}\left[\varphi_{a}\right]_{C^{\alpha},[0,1]}\left[A_{I_{n}}\right]_{\mathrm{Lip}}^{\alpha}=\left|I_{n}\right|^{1-\alpha}\left[\varphi_{a}\right]_{C^{\alpha},[0,1]}
$$

and a similar estimate holds for $\left[\psi_{a}^{-1}\right]_{C^{\alpha}, I_{n}}$. Therefore, observing that for each $a \in(0,1]$, we have $\psi_{a} \mid \partial I_{n} \equiv \mathrm{id}$, it follows that we may apply Proposition 2.1(ii). Hence, for all $\alpha \leq a, \psi_{a}$ is a bi- $\alpha$-Hölder homeomorphism. Since $f_{a}=\psi_{a}^{-1} \circ f_{1} \circ \psi_{a}$ and $f_{1}$ preserves Lebesgue measure $\mu$, it follows that the pullback $\psi_{a}^{*} \mu$ is an invariant measure for $f_{a}$. As the functions $\varphi_{a}$ are absolutely continuous with respect to Lebesgue measure, it follows that $\psi_{a}$ is also absolutely continuous.

Proof of Theorem A (Transitivity). Finally, we show how to modify $f_{a}$ so that the resulting maps are transitive. We actually embed $f_{a}$ into a continuous family $f_{a, \varepsilon}, \varepsilon \in\left(0, \frac{1}{2}\right)$, where $f_{a, \varepsilon}$ is transitive for $\varepsilon \neq 0$.

We modify the model maps $g_{a, b}$ as follows. Let

$$
g_{a, b, \varepsilon}=A_{[-\varepsilon, 1+\varepsilon]}^{-1} \circ g_{a, b}, \quad g_{a, b, \varepsilon}^{-}=A_{[-\varepsilon, 1]}^{-1} \circ g_{a, b} .
$$

Define $f_{a, \varepsilon}$ by

$$
f_{a, \varepsilon}(x)=\left\{\begin{array}{ll}
A_{I_{1}}^{-1} \circ g_{a, b, \varepsilon}^{-} \circ A_{I_{1}}(x) & x \in I_{1} \\
A_{I_{n}}^{-1} \circ g_{a, b, \varepsilon} \circ A_{I_{n}} & x \in I_{n}, n=2,3, \ldots . \\
0 & x=0
\end{array} .\right.
$$

(using that for each interval $J, A_{J}$ extend affinely to $\mathbb{R}$ ). Note that we have modified $f_{a}$ on the interval $I_{1}$ differently to the other intervals $I_{n}, n=2,3, \ldots$, to ensure that $f([0,1])=[0,1]$. It is clear from the preceding arguments that $f_{a, \varepsilon}$ satisfies properties (1)-(3) of Theorem A. It just remains to show transitivity.

Observe that the intervals $I_{1}, I_{2}, \ldots$ are no longer invariant. The interval $I_{1}$ contains a single maximal open interval $G_{1,1, a, \varepsilon}=G_{1,1}$ for which $f_{a, \varepsilon}\left(G_{1,1}\right) \subset I_{2}$ and, in fact, $f_{a, \varepsilon}\left(\bar{G}_{1,1}\right)$ is a closed neighbourhood of the repelling fixed point $2^{-1}$. For $n=2,3, \ldots$ we have that

- the interval $I_{n}$ contains pairwise disjoint maximal open intervals

$$
G_{n, 1,-}, G_{n, 2,-}, \ldots, G_{n, n,-}
$$

such that, for $k=1,2, \ldots, n, f_{a, \varepsilon}\left(G_{n, k,-}\right) \subset I_{n-1}$ and, in fact, $f_{a, \varepsilon}\left(\bar{G}_{n, k,-}\right)$ is a closed right-neighbourhood of the repelling fixed point $2^{-n+1}$.

- the interval $I_{n}$ contains pairwise disjoint maximal open intervals

$$
G_{n, 1,+}, G_{n, 2,+}, \ldots, G_{n, n,+}
$$

such that, for $k=1,2, \ldots, n, f_{a, \varepsilon}\left(G_{n, k,+}\right) \subset I_{n+1}$ and, in fact, $f_{a, \varepsilon}\left(\bar{G}_{n, k,+}\right)$ is a closed left-neighbourhood of the repelling fixed point $2^{-n}$. 
The key property of the maps $g_{a, b, \varepsilon}$ that we will now use is the following: for any fixed point $x$, for any closed neighbourhood $V$ of $x$ there exists a positive integer $\ell$ and a closed neighbourhood $U \subset V$ of $x$ such that $g_{a, b, \varepsilon}^{\ell}(U)=[0,1]$. This property implies that for any positive integers $m$ and $n$, for neighbourhoods $U$ in $I_{n}$ and $V$ in $I_{m}$ there exists a positive integer $r$ such that $f_{a, \varepsilon}^{r}(U) \cap V$. Hence $f_{a, \varepsilon}$ is topologically transitive.

For $a \neq 1$, the above analysis can also be applied to the construction when restricting to the union of the intervals $I_{n}, I_{n+1}, \ldots$, just as in the case of Corollary 2.1. Thus, analogously to that corollary, we also get the following result.

Corollary 2.3. For each $a \in(0,1)$, there exists a sequence $f_{a, n} \in C^{0}([0,1],[0,1])$ satisfying properties $3(a)-3(c)$ in Theorem $A$ above and with the additional property that $\lim _{n \rightarrow \infty} f_{n}=\mathrm{id}$ where convergence is taken

- in the $C^{\alpha}$-topology for any $\alpha \in(0, a)$,

- in the $W^{1, p}$-topology for any $p \in\left[1,(1-a)^{-1}\right)$.

\section{Examples in the little Zygmund class}

As was previously remarked, the function $f_{1}$ constructed in Theorem A above has modulus of continuity $t \log \left(\frac{1}{t}\right)$, and hence is $\alpha$-Hölder for every $\alpha \in[0,1)$, but it is not Lipschitz. Further, it does not satisfy either the big or little Zygmund conditions. Recall that a continuous function $f$ of the interval $[0,1]$ satisfies the big Zygmund condition if, for all $x$ in $(0,1)$,

$$
|f(x+t)+f(x-t)-2 f(x)|=O(t)
$$

and the little Zygmund condition if, for all $x$ in $(0,1)$,

$$
|f(x+t)+f(x-t)-2 f(x)|=o(t) .
$$

Observe that this condition only makes sense at interior points. We will denote the sets of functions satisfying the big and little Zygmund conditions respectively by $C^{Z}([0,1], \mathbb{R})$ and $C^{z}([0,1], \mathbb{R})$. Observe that these are both linear spaces. Define the Zygmund semi-norm by

$$
[f]_{Z,[0,1]}=\sup _{t: x \pm t \in[0,1]} \sup _{x \in[0,1]} \frac{|f(x+t)+f(x-t)-2 f(x)|}{|t|} .
$$

Then

$$
\|f\|_{C^{Z}([0,1], \mathbb{R})}=\|f\|_{C^{0}([0,1], \mathbb{R})}+[f]_{Z,[0,1]}
$$


defines a complete norm on $C^{Z}([0,1], \mathbb{R})$, which we call the Zygmund norm. With this topology, $C^{z}([0,1], \mathbb{R})$ is a closed subspace of $C^{Z}([0,1], \mathbb{R})$.

The Zygmund classes strictly (in fact compactly) contain the Lipschitz class and are contained in the $\alpha$-Hölder class for each $\alpha \in[0,1)$. Moreover, by a theorem of Zygmund [19], functions in the big Zygmund class have modulus of continuity $t \log \left(\frac{1}{t}\right)$. (See [19] for more on these classes.) The reason that the example $f$ given above is not in either Zygmund class is that, at a turning point $x$ of a $b$-branched horseshoe, for all $t$ sufficiently small,

$$
|(f(x+t)-f(x))-(f(x)-f(x-t))| \geq 2 b t .
$$

One might think that, by replacing piecewise-affine with smooth horseshoes and possibly changing the lengths of the intervals, one may be able to improve the regularity, say to the big Zygmund class. However, this is not possible as, for any $b$-branched horseshoe, there is some turning point $x$ and some $t$ so that

$$
|(f(x+t)-f(x))-(f(x)-f(x-t))| \geq b t .
$$

(To see this, let $I_{b}$ be the domain of the horseshoe and take the pairwise disjoint subintervals $I_{b, 1}, I_{b, 2}, \ldots, I_{b, b}$ of maximal size on which $f$ is monotone. Take $I_{b, k}$ of minimal length. Let $x$ be either of the endpoints of $I_{b, k}$. Let $I_{b, \ell}$ be the other interval sharing $x$ as an endpoint. Let $t=\left|I_{b, k}\right|$. Since $f$ changes from monotone increasing to monotone decreasing or vice versa going from $I_{b, k}$ to $I_{b, \ell}$, we have $\mid(f(x+t)-f(x))-$ $(f(x)-f(x-t))|\geq \max \{|f(x+t)-f(x)|,|f(x)-f(x-t)|\} \geq| I_{b}|\geq b| I_{b, k} \mid=b t$. The last inequality follows as $I_{b, k}$ was chosen to have minimal length.)

Remark 3.1. It will be clear from the construction below that piecewise-affine (with countably many pieces, as in the preceding example) can also be constructed but, necessarily, these can only lie in the big Zygmund class.

Before describing the construction, we introduce the following notation. Given oriented closed intervals $I$ and $J$ denote by $A_{I, J}$ the unique orientation-preserving affine bijection from $J$ to $I$, and $A_{I, J}^{-}$the unique orientation-reversing affine bijection from $J$ to $I$. Given a positive integer $n$, let $g_{n}:[0,1] \rightarrow\left[0, \frac{1}{n}\right]$ be defined by

$$
g_{n}(x)=\frac{1}{2 n}(1-\cos (2 \pi n x)) .
$$

Observe that, for each $n, g_{n}$ maps the interval $[0,1]$ onto $\left[0, \frac{1}{n}\right]$ in a $2 n$-to- 1 manner. Also, $g_{n}$ is Lipschitz with Lipschitz constant $\pi$, and is differentiable with vanishing derivative at the endpoints. It also has the following important property.

Key Property: Let $\phi:\left[0, \frac{1}{n}\right] \rightarrow[0,1]$ be any homeomorphism. Then the composition $\phi \circ g_{n}:[0,1] \rightarrow[0,1]$ is a $2 n$-branched horseshoe. 

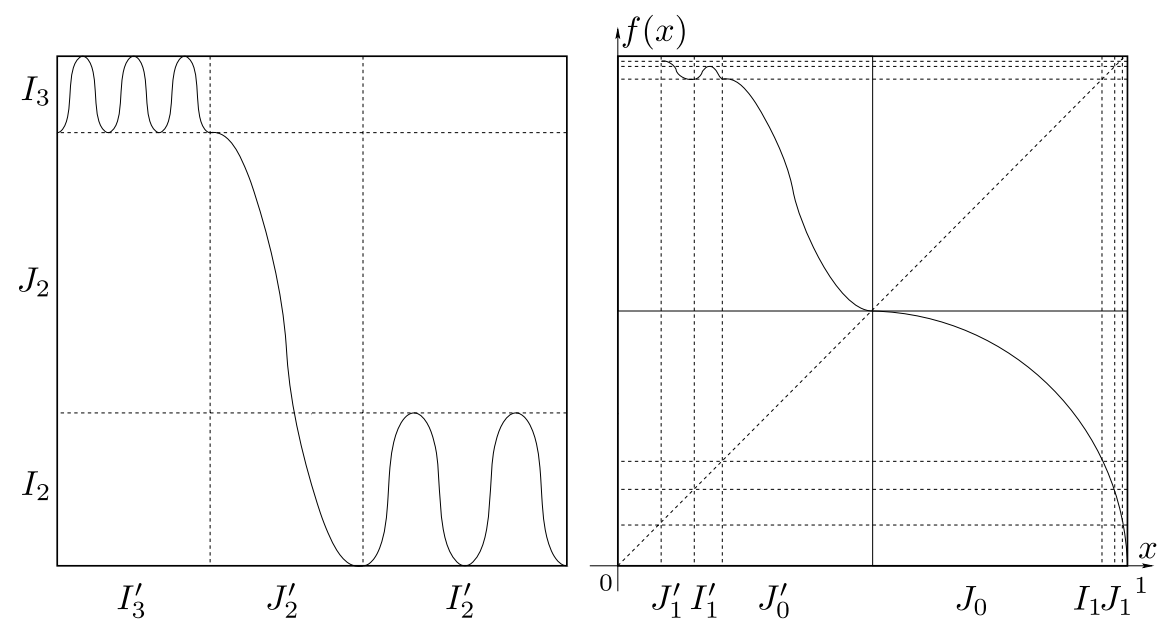

Figure 2. The graph of the little Zygmund interval endomorphism $f$ on $[0,1]$ with infinite topological entropy (right) and an affine rescaling of the graph on the interval $I_{3}^{\prime} \cup J_{3}^{\prime} \cup I_{2}^{\prime}$ (left).

The construction is now the following. Take any continuous map $f:\left[\frac{1}{2}, 1\right] \rightarrow$ $\left[0, \frac{1}{2}\right]$ satisfying the following properties

1. $f$ is an orientation-reversing homeomorphism which is little Zygmund, with little Zygmund inverse,

2. $f^{\prime}\left(\frac{1}{2}\right)$ exists and is zero,

3. $f^{\prime}(1)$ exists (in the extended sense) and equals $-\infty$,

4. There exist pairwise disjoint open subintervals $I_{1}, I_{2}, \ldots \subset\left[\frac{1}{2}, 1\right]$, ordered from left to right, which converge to $\{1\}$ and have the property that, for all $n \in \mathbb{N}$,

$$
\frac{\left|f\left(I_{n}\right)\right|}{\left|I_{n}\right|}=n .
$$

5. Let $J_{0}, J_{1}, J_{2}, \ldots \subset[0,1]$ denote the pairwise disjoint closed intervals which are connected components of $\left[\frac{1}{2}, 1\right] \backslash \bigcup_{n=1}^{\infty} I_{n}$, again ordered from left to right. Then, there exists a positive real number $K$ so that, for all $n \in \mathbb{N}$,

$$
\frac{\left|I_{n}\right|+\left|J_{n}\right|}{\left|f\left(J_{n}\right)\right|} \leq K .
$$

Remark 3.2. Observe that such functions are easy to construct. For example, if we start with the function $\phi:\left[0, \frac{1}{e}\right] \rightarrow\left[0, \frac{1}{e}\right]$ given by $\phi(x)=x \log \left(\frac{1}{x}\right)$, then $\phi$ has infinite derivative at zero, a critical point at $\frac{1}{e}$, and satisfies the Zygmund condition. Moreover, so do any affine rescalings. Thus, setting

$$
f=A_{\left[0, \frac{1}{e}\right],\left[0, \frac{1}{2}\right]} \circ \phi \circ A_{\left[\frac{1}{2}, 1\right],\left[0, \frac{1}{e}\right]}^{-},
$$


we find that $f$ satisfies properties (1)-(3). We can choose intervals $I_{n}$ to be a small neighbourhood about the unique point $x_{n}$ where $f^{\prime}\left(x_{n}\right)=-n$, for each $n$, so that (4) is satisfied. By taking $\left|I_{n}\right|$ sufficiently small for each $n$, this will ensure that $\frac{\left|I_{n}\right|+\left|J_{n}\right|}{\left|f\left(J_{n}\right)\right|} \leq 2 \frac{\left|J_{n}\right|}{\left|f\left(J_{n}\right)\right|}$. As $\left|f^{\prime}(y)\right|$ tends to infinity as $y$ tends to 1 , the property (5) holds.

Next, extend $f$ to a continuous map from $[0,1]$ to $[0,1]$ as follows. First, however, we need the following notation. Recall that $\nu(x)=1-x$ is the unique affine orientation-reversing map of $[0,1]$. Define $\sigma:[0,1] \rightarrow[0,1]$ by

$$
\sigma(x)=\frac{1}{2}(1-\cos (\pi x)) \text {. }
$$

(More generally, $\sigma$ can be any smooth orientation-preserving homeomorphism of $[0,1]$ with bounded derivative and with critical points at 0 and 1.)

For each $n$, define $I_{n}^{\prime}=f\left(I_{n}\right)$ and $J_{n}^{\prime}=f\left(I_{n}\right)$. Observe that, since $\left.f\right|_{\left[\frac{1}{2}, 1\right]}$ is an orientation-reversing homeomorphism, the $I_{n}^{\prime}$ form a decreasing sequence of pairwise disjoint subintervals, converging to $\{0\}$, and are interlaced by the $J_{n}^{\prime}$. We define $f$ on each $I_{n}^{\prime}$ and $J_{n}^{\prime}$ separately, ensuring that the resulting map is continuous. First, define $f$ on $I_{n}^{\prime}$, for each integer $n \geq 1$, by

$$
f(x)=A_{\left[0, \frac{1}{n}\right], I_{n}} \circ g_{n} \circ A_{I_{n}^{\prime},[0,1]}(x) \quad x \in I_{n}^{\prime}, n \geq 1 .
$$

Observe that $f$ is differentiable on each $I_{n}^{\prime}$ and has vanishing derivative at the endpoints. Define $f$ on $\bigcup_{n=0}^{\infty} J_{n}^{\prime}$ so that $f$ is differentiable on each $J_{n}^{\prime}$ with vanishing derivatives at the endpoints. Namely, we set

$$
f(x)=\left\{\begin{array}{ll}
A_{[0,1], I_{n} \cup J_{n}} \circ \nu \circ \sigma \circ A_{J_{n}^{\prime},[0,1]}(x) & x \in J_{n}^{\prime}, n \geq 1 \\
A_{[0,1], J_{0}} \circ \nu \circ \sigma \circ A_{J_{0}^{\prime},[0,1]}(x) & x \in J_{0}^{\prime}
\end{array} .\right.
$$

Proof of Theorem B. Observe that $\left.f\right|_{I_{n}^{\prime}}$ are differentiable with uniformly bounded derivative over all $n$. In fact, for all $n \in \mathbb{N}$,

$$
\left\|f^{\prime}\right\|_{C^{0}\left(I_{n}^{\prime}, \mathbb{R}\right)} \leq \pi
$$

Also the $\left.f\right|_{J_{n}^{\prime}}$ are differentiable and, by inequality (3.9) above, the derivatives are also uniformly bounded over all $n \neq 0$ since, for all integers $n \geq 1$,

$$
\left\|f^{\prime}\right\|_{C^{0}\left(J_{n}^{\prime}, \mathbb{R}\right)}=\frac{\left|I_{n} \cup J_{n}\right|}{\left|J_{n}^{\prime}\right|}\left\|\sigma^{\prime}\right\|_{C^{0}([0,1], \mathbb{R})} \leq K \pi / 2 .
$$

Since $f \mid J_{0}$ is also differentiable, and on all intervals $I_{n}^{\prime}$ and $J_{n}^{\prime}, f^{\prime}$ vanishes at the endpoints, it follows that $\left.f\right|_{\left(0, \frac{1}{2}\right]}$ is also differentiable. Hence $\left.f\right|_{\left[0, \frac{1}{2}\right]}$ is little Zygmund. Next, as $\left.f\right|_{\left[\frac{1}{2}, 1\right]}$ is little Zygmund and $f$ is differentiable from the left- 
and from the right at $x=\frac{1}{2}$ (with zero derivative), it follows that $f$ is little Zygmund on $[0,1]$.

Let us now show that $f$ has infinite topological entropy. Since $f\left(I_{n}\right)=I_{n}^{\prime}$ and $f\left(I_{n}^{\prime}\right)=I_{n}$ it follows that $I_{n}^{\prime}$ is $f^{2}$-invariant, for each $n$. Moreover $\left.f\right|_{I_{n}}$ is a homeomorphism from $I_{n}$ to $I_{n}^{\prime}$, and $\left.f\right|_{I_{n}^{\prime}}$ is an affine rescaling of $g_{n}$. Thus by the Key Property stated above, $\left.f^{2}\right|_{I_{n}}$ is a $2 n$-branched horseshoe. Hence $\left.f^{2}\right|_{I_{n}}$ has topological entropy at least $\log (2 n)$. Consequently

$$
h_{\mathrm{top}}\left(f^{2}\right) \geq \sup _{n} h_{\mathrm{top}}\left(\left.f^{2}\right|_{I_{n}^{\prime}}\right)=\sup _{n} \log (2 n)=\infty .
$$

Finally, since it is known that for an arbitrary continuous self-map $F$ of a compact metric space the equality $h_{\text {top }}\left(F^{k}\right)=k h_{\text {top }}(F)$ holds for any positive integer $k$, it follows that $h_{\text {top }}(f)=+\infty$ as well.

\section{Concluding remarks}

We finish with a number of open problems suggested by this work.

1. Do there exist one-dimensional endomorphisms with infinite topological entropy which are asymptotically $h$-expansive, or even $h$-expansive? What is their 'optimal' regularity: can they be Hölder or Sobolev?

2. The Zygmund example has the property that its second iterate is no longer Zygmund. (It is of the type given in Theorem A which, as remarked on in Section 3 cannot lie in the Zygmund class.) Does there exist a map in the (big or little) Zygmund class with infinite topological entropy, and such that all iterates are also in the (big or little) Zygmund class?

3. (Alby Fisher) Is there an ergodic example of a map with infinite entropy in dimension-one? Within some Hölder, Sobolev or Zygmund class?

Acknowledgements. The author would like to thank IME-USP, ICERM (Brown University), Uppsala University and KTH Stockholm for their hospitality. I would also like to thank E. de Faria, C. Tresser and M. Benedicks for many stimulating conversations regarding the relation between regularity and entropy. I also thank M. Benedicks for the question that lead to this work. Finally, I thank the referee for their interesting comments and questions.

\section{References}

1. Adler, R. L., Konheim, A. G. and MacAndrew, M. H., Topological entropy, Trans. Amer. Math. Soc. 114 (1965), 309-319. MR0175106 
2. Boroński, J. P., Kupka, J. and Oprocha, P., All minimal Cantor systems are slow, Bull. Lond. Math. Soc. 00 (2019), 1-8. MR4041001

3. Bowen, R., Entropy for group endomorphisms and homogeneous spaces, Trans. Amer. Math. Soc. 153 (1971), 401-414. MR0274707

4. Bowen, R., Entropy-expansive maps, Trans. Amer. Math. Soc. 164 (1972), 323331. MR0285689

5. de Faria, E., Hazard, P. and Tresser, C., Genericity of infinite entropy for maps with low regularity, 2017. ArXiv preprint. arXiv : 1709. 02431.

6. de Faria, E., Hazard, P. and Tresser, C., Infinite entropy is generic in Hölder and Sobolev Spaces, C. R. Acad. Sci. Paris, Ser. I 355 (2017), 11851189. MR3724883

7. Denker, M., Grillenberger, Ch. and Sigmund, K., Ergodic Theory on Compact Spaces, Lecture Notes in Math. 527, Springer, Berlin-Heidelberg-New York, 1976. MR0457675

8. Evans, L. C. and Gariepy, R. F., Measure Theory and Fine Properties of Functions, Revised ed., CRC Press, Taylor \& Francis Group, Chapman \& Hall, 2015. MR3409135

9. Gambaudo, J.-M. and Martens, M., Algebraic topology for minimal Cantor sets, Ann. Henri Poincaré 7 (2006), 423-446. MR2226743

10. Grillenberger, C., Constructions of strictly ergodic systems, Z. Wahrsch. Verw. Gebiete 25 (1973), 323-334. MR0340545

11. Іто, S., An estimate from above for the entropy and topological entropy of a $C^{1}$-diffeomorphism, Proc. Japanese Acad. Sci. 46 (1970), 226-230. MR0272981

12. Katok, A. and Hasselblatt, B., Introduction to the Modern Theory of Dynamical Systems, Encyclopedia of Mathematics and Its Applications 54, Cambridge University Press, 1995. MR1326374

13. Misiurewicz, M., Topological conditional entropy, Studia Math. LV.2 (1976), 175200. MR0415587

14. Misiurewicz, M., Horseshoes for continuous mappings of an interval, in Dynamical Systems, CIME Summer Schools 78, pp. 125-135, Springer, 2011. MR0660643

15. Pugh, C. C., Real Mathematical Analysis, 1st ed., University Texts in Mathematics, Springer, 2002. MR1886084

16. Whlters, P., An Introduction to Ergodic Theory, Graduate Texts in Mathematics 79, Springer, 1982. MR0648108

17. YAnO, K., A remark on the topological entropy of homeomorphisms, Invent. Math. 59 (1980), 215-220. MR0579700

18. Ziemer, W., Weakly Differentiable Functions, Graduate Texts in Mathematics 120, Springer, 1989. MR1014685

19. Zygmund, A., Trigonometric Series, 3rd. Ed., Vols. I \& II combined (with a foreword by Robert Fefferman). Cambridge Mathematical Library, Cambridge University Press, 2002. MR1963498 
Peter Hazard

Instituto de Matemática e Estatística

Universidade Federal Fluminense

Niterói, RJ

Brazil

peterh@id.uff.br

Received November 3, 2017

in revised form September 13, 2019 